\title{
PVC/PE 共混物中的增容 - 交联协同作用的研究*
}

文献中采用单一的增容或交联方法以改 善 PVC/PE 的相容性, 早有报道 ${ }^{[1,2]}$. 我们 $]^{[3 \sim 5]}$ 则用增容 - 交联并用的方法可显著提高 PVC/LDPE 共混物的力学性能, 并在此基础 上提出了增容 - 交联协同的设想. 本文又考 察了增容 - 交联并用技术对 PVC/HDPE 体 系的作用。

实验所用聚氯乙烯 $(\mathrm{PVC})$ 为萧山树脂厂 生产的二型树脂; 高密度聚乙烯 (HDPE) 为 齐鲁不化总公司生产的 (DMD6158)；增容剂 为氯化聚乙烯 (CPE, 含氯 $35 \%)$; 交联剂为 过氧化二异丙苯 $(\mathrm{DCP}) /$ 氯化镁 $(\mathrm{MgO}) /$ 三 烯丙基异氮尿酸酯 (TAIC) $(2 / 0.5 / 1.5$, 重量比) 复合体系. 制样和性能测试方法同文献[3].

PVC $/ H D P E(50 / 50$, 重量比) 共混物的力 学性能极差, 其拉伸强度仅 $1.7 \mathrm{MPa}$, 断裂伸 长率仅 $10 \%$. 在 $100 \mathrm{~g}$ 共混物中加 $5 \mathrm{~g}$ 增容 剂, 这两项指标分别提高至 $16.7 \mathrm{MPa}$ 和 $27 \%$. 而单加 $4 \mathrm{~g}$ 交联剂, 则分别提高至 $16.2 \mathrm{MPa}$ 和 $77 \%$ ，这两个体系的性能虽然比简单共 混物明显提高, 但仍属脆性材料. 若将 $5 \mathrm{~g}$ 增容剂和 $4 \mathrm{~g}$ 交联剂一并加人, 则拉伸强度 提高至 17.1MPa, 断裂伸长率则提高至 $240 \%$, 拉伸时材料明鼠为韧性断裂行为, 这表明增 容 - 交联并用技术对 PVC/HDPE 体系同样 有效。

为进一步研究增容 - 交联协同的机理, 本文进行了抽提测试. 样品置于索氏抽提器 中, 用四氢呋喃作溶剂, 在浴温 $100^{\circ} \mathrm{C}$ 下抽提 $50 \mathrm{~h} . \mathrm{PVC} / \mathrm{HDPE}(50 / 50)$ 共混物抽提后剩余 的不溶物重量为样品总重的 $44.0 \%$. 加 $4 \mathrm{~g}$ 交联剂后, 不溶物比例为 $45.8 \%$, 提高极有 限, 说明此时很少有 PVC 与 HDPE 的互交 联产物生成。在此体系中再加入 $5 \mathrm{~g}$ 增容剂 厉，剩余的不溶物比例升至 $75.7 \%$, 表明大量
PVC 参与交联, 从而由可溶变为不可溶.

由以上结果可知, 在不相容聚合物共混 物中, 单加增容剂虽能提高两相分散程度, 但因相间粘结仍靠范德华力, 故对力学性能 的改善有限。两组份的互交联能提高相间粘 结力, 但加交联剂时, 由于 DCP 较多地集中 在 HDPE 相, 而且两相分散程度低, 故互交 联产物少, 改性效果不显著. 而当增容剂和 交联剂同时加人时,增容剂促进了两组分的 相互分散, 增加了两组分的相互接触, 减少 了相尺寸,而交联剂出现在两相间的儿率也 大大提高, 致使两组分间产生大量互交联 物, 从而显著提高了相间粘结力, 由于这种 增容 - 交联协同作用, 使共混物的力学性能 显著提高.

\section{参考文献}

1 Paul D R, Locke C E, Vinson C E. Chlorinated polyethylene modification of blends dreived from waste plastics. part 1: mechinical bchavior. Polym Eng Sci. 1973, 13:202

2 Nakamura Y, Mori K, Nishira $\mathrm{H}$ et al. Cocrosslinking blends of polyethylene and poly (vinyl chloride). Kobunshi Ronbunshu, 1985, 42: 567

3 许承威, 鲍诗杰. 不相容聚合物共混中增容 - 交联协 同作用研究. 中国塑料, 1991, 5(2): 43

4 Xu Chengwei, Fang Zhengping, Zhong Jianhua. Study on compatibilization-crosslinking synergism in PVC/PE blends. Angew Makromol Chem, 1993. 212: 45

$5 \mathrm{Xu}$ Chengwei, Zhong Jianhua, Fang Zhengping. Influence of NBR on compatibility between PVC and LDPE and its synergism with crosslinking agent. Proceedings of 34th IUPAC Congress, Beijing China, 15 20 Aug. 1993

方征平许承威 ** 鲍诗杰赵益飞 (杭州大学化学系, 杭州 310028)

*国家白然科学基金资助项 H

**联系人 